\title{
La gestion par la dérogation : une politique d'apprentissage industriel en Belgique francophone
}

Par Audrey Levêque et Didier Vrancken*

Ce dispositif de formation en alternance s'adresse prioritairement aux jeunes de quinze à dix-huit ans, en difficultés scolaires. Sur le terrain, la branche professionnelle se réapproprie cette politique d'emploi. L'apprentissage industriel concerne des diplômés âgés de plus de vingt ans.

Le début des années 1980 est marqué par une montée massive du chômage et des «formes particulières d'emploi » (Maruani et Reynaud, 1993), ainsi que des contrats atypiques touchant particulièrement les jeunes peu qualifiés. La remise en question progressive de la société salariale s'est traduite par une fragilisation des contrats à durée indéterminée et à temps plein, une flexibilité croissante des formes d'intégration au marché du travail, un développement du sousemploi et un recours accru des entreprises aux temps partiels, aux contrats à durée déterminée et aux formes intérimaires de l'emploi (ministère de l'Emploi et du Travail, 2000).

Dans un contexte d'expansion de phénomènes de précarisation, de désaffiliation ${ }^{1}$ (Castel, 1995), de disqualification sociale (Paugam, 1991) et de montée de nouvelles inégalités sociales (Rosanvallon et

${ }^{1}$ Effet de l'absence de participation à toute activité productive et de l'isolement relationnel.
* Audrey Levêque est sociologue, aspirant du Fonds national de la recherche scientifique (FNRS). Elle fait partie du Centre de recherches et d'intervention sociologiques (CRIS) de l'université de Liège en Belgique et du Groupe d'analyse des politiques publiques (GAPP) de L'École normale supérieure de Cachan (ENS). Elle travaille sur les politiques publiques de formation-emploi pour les jeunes.

Didier Vrancken est professeur de sociologie au Service de sociologie des organisations et de l'intervention, et directeur du Centre de recherches et d'intervention sociologiques (CRIS) au Département de sciences sociales de I'université de Liège. II travaille sur les politiques sociales. Il a publié, en 2001, avec O. Kuty, La sociologie et l'intervention. Enjeux et perspectives, De Boeck Université. Son dernier ouvrage, paru aux Éditions Labor, a pour titre Le crépuscule du social.

Courriel : Audrey.Leveque@ulg.ac.be et

Didier.Vrancken@ulg.ac.be 
Fitoussi, 1996), nombre d'observateurs se sont penchés sur la résurgence d'une «nouvelle question sociale »(Rosanvallon, 1995). Après la crise des années 1970 et les critiques de l'État-providence qui en découlèrent, les années 1980 se caractérisent par une remise en cause des politiques perçues comme impersonnelles, passives, indemnisatrices et grevant lourdement le budget de l'État (Rosanvallon, 1981). À la critique néolibérale dirigée vers les fondements

\section{Encadré 1 \\ La Fédé}

La Fédé est une fédération patronale belge constituée en association de fait en 1906 et transformée en ASBL (Association sans but lucratif) en 1946. Elle regroupe plus de 1000 entreprises et représentait, en 1999, 211300 emplois. Diverses raisons nous ont poussés à choisir ce secteur pour l'étude de l'apprentissage industriel.

Premièrement, ce secteur est un des plus importants en Wallonie en matière d'emplois (Brion et Moreau, 1996) puisqu'il représente environ un tiers de l'emploi industriel. Deuxièmement, La Fédé s'est préoccupée de formation très tôt dans son histoire et la formation en alternance y a bénéficié d'une place non négligeable. Troisièmement, ce secteur est un des plus impliqués dans l'apprentissage industriel (Mariën, 1997). Aujourd'hui encore, en Belgique, près de la moitié des contrats d'apprentissage industriel (CAll sont signés par des entreprises membres de La Fédé. Enfin, dans ce secteur, le taux d'engagement des apprentis en fin de formation est très élevé puisqu'il atteint $90 \%$ (IFPM $\left.{ }^{*}\right)$ 1999).

L'apprentissage industriel est une compétence fédérale qui relève du ministère de l'Emploi et du Travail. D'emblée, nous pouvons donc noter que cette matière n'est pas issue de la sphère de l'éducation mais bien de celle du travail.

${ }^{(*)}$ Institut de formation postscolaire de la branche professionnelle étudiée. même de l'État-providence succéda une critique misant sur la débureaucratisation de l'intervention étatique et sur la dynamisation de la société civile. Ces deux critiques convergeant, elles encouragèrent un mouvement de transformation des modalités de l'intervention étatique. En Belgique, de nouvelles politiques sociales voient le jour; elles contribuent à la superposition d'une nouvelle figure extrêmement ambiguë de l'État (Giovannini, 2000), celle de « l'État-social actif ». Elles visent progressivement les populations les plus fragiles en tant que cibles prioritaires ; il s'agit de les « activer» dans le circuit de l'emploi, en relevant le taux d'activité et en garantissant par là l'avenir de la sécurité sociale. On peut aujourd'hui postuler que la démultiplication des emplois aidés a contribué à cibler les plus faibles et à les fragiliser davantage à travers le développement et le maintien d'emplois précaires (Paugam, 2000); ceux-ci conduisant au renforcement d'une véritable zone d'emplois atypiques ou de sous-emploi sur un marché du travail que l'on sait fortement dualisé (Berger et Piore, 1980 ; Paradeise, 1987).

À partir d'une étude menée en Belgique, dans le secteur de la formation en alternance, nous tenterons d'illustrer en quoi les acteurs les plus qualifiés profitent prioritairement de ces politiques. En recourant systématiquement à la dérogation, les entreprises affiliées à une association patronale, La Fédé (cf. encadré 1), parviennent à embaucher des jeunes plus âgés, mieux intégrés socialement et familialement que le public ciblé initialement par la loi sur l'apprentissage industriel. Une véritable régulation par la dérogation des contrats d'apprentissage industriel ( $c f$. encadré 2) se met alors en place ; elle conduit à la sélection de ces jeunes et au maintien de l'exclusion des moins qualifiés. Ce dispositif public ciblant des jeunes vise bien, au départ, une diminution globale du chômage ; toutefois, on peut retenir qu'il peut se voir détourné de sa mission sociale d'insertion à des fins de flexibilité de la main-d'œuvre. Pour l'entreprise en effet, recruter un jeune sur la base d'un emploi temporaire permet de le mettre à l'épreuve, d'évaluer les compétences nécessaires et de le sélectionner. Mais, ce faisant, cette pratique maintient les moins qualifiés dans le sous-emploi.

\footnotetext{
${ }^{2}$ Afin de respecter la règle de confidentialité, nous avons opté pour une modification de l'appellation de l'association patronale.
} 


\section{Encadré 2 \\ Le contrat d'apprentissage industriel}

L'apprentissage industriel (*) est une formation en alternance par laquelle un jeune apprend sur le terrain une profession qu'il a choisie tout en poursuivant une formation théorique, générale et professionnelle, dans un Centre d'éducation et de formation en alternance (CEFA). Ce dispositif s'adresse aux jeunes à partir de 15/16 ans et jusqu'à 18 ans, soumis à l'obligation scolaire à temps partiel (cf. encadré 3). Mais des dérogations sont toujours possibles jusqu'à 21 ans et parfois au-delà. II s'agit d'offrir aux jeunes qui ne peuvent ou ne veulent pas suivre les filières classiques de l'enseignement une formation professionnelle complète afin d'acquérir ainsi une expérience.

Le Contrat d'apprentissage industriel (CAll) est à durée déterminée. L'employeur s'engage à fournir à l'apprenti une formation en vue de l'exercice de la profession choisie. L'apprenti est obligé d'acquérir, sous l'autorité de l'employeur, la pratique de la profession et de suivre les cours nécessaires. La durée de l'apprentissage est fixée par le règlement d'apprentissage avec un minimum de six mois.

La mise en place de l'apprentissage industriel est à l'initiative des Commissions paritaires des différents secteurs (représentation des travailleurs et des employeurs). Celles-ci instaurent un Comité paritaire d'apprentissage (CPA) dont le secrétaire est issu du ministère du Travail. Syndicats des travailleurs et des employeurs gèrent ainsi ensemble l'apprentissage industriel. Le CPA établit le règlement d'apprentissage, le modèle de programme de formation, organise les différentes épreuves de fin de formation et décide de la répartition du temps entre formation pratique et théorique.

La loi permet à ces comités de déroger à la limite d'âge de 18 ans. Les conditions de cette dérogation sont précisées dans le règlement d'apprentissage de chaque CPA. Pour la branche professionnelle étudiée, "La règle générale veut qu'un apprenti puisse conclure un contrat d'apprentissage à partir de 15 ans jusqu'à 17 ans. Le CPA peut déroger à cette règle pour les jeunes de 18 à 20 ans. Dans les cas exceptionnels, le CPA peut également déroger à cette règle pour les jeunes de 21 ans et plus qui souhaitent conclure un contrat d'apprentissage industriel ». En pratique, toute demande de dérogation est acceptée par le comité. Ainsi, certains jeunes terminent leur apprentissage à 25 ans.

Tout au long du processus, l'apprenti est considéré comme un travailleur ordinaire à temps partiel. ॥ perçoit une indemnité mensuelle d'apprentissage qui correspond à un pourcentage du revenu mensuel moyen minimum garanti national (1 117,88 € brut).

En janvier 2000, une enquête du Fond social européen (FSE) estimait à 401 le nombre de contrats d'apprentissage conclus en Communauté française de Belgique, toutes branches professionnelles confondues. Le groupe cible direct de ce dispositif représentait 5415 jeunes des Centres d'éducation et de formation en alternance assurant l'enseignement à horaire réduit (obligation scolaire à temps partiel). Ce nombre reste en-deçà de l'utilisation d'autres dispositifs jeunes en Belgique francophone. En effet, si nous le comparons à deux autres mesures proches de l'apprentissage (alternance également) et s'adressant aux jeunes de 15 à 18 ans, nous pouvons noter que, d'une part, la Convention d'insertion socioprofessionnelle (CISP) concernait 2704 jeunes et d'autre part, parallèlement aux CEFA et répondant à I'obligation scolaire à temps partiel également, l'apprentissage des Classes moyennes comptait 7453 jeunes. Ce faible nombre de contrats d'apprentissage industriel peut s'expliquer par la grande méconnaissance du dispositif de la part de beaucoup d'employeurs mais aussi par le coût, parfois dissuasif, de ce type de formation. En effet, comme nous le verrons, les jeunes reçoivent une rémunération relativement conséquente par rapport à ce que permettent les autres types de mesures jeunes.

${ }^{(*)}$ Loi du 19 juillet 1983 sur l'apprentissage de professions exercées par des travailleurs salariés, modifiée par les lois du 24.07.87, 20.07.92, 13.02 .98 et 6.05 .98 . 


\section{Encadré 3 \\ Le système d'enseignement en Communauté française de Belgique}

\begin{tabular}{|c|c|c|c|c|}
\hline \multirow{3}{*}{$\begin{array}{l}\text { Obligation } \\
\text { scolaire } \\
\text { à temps plein }\end{array}$} & De 6 à 12 ans & $\begin{array}{c}\text { Enseignement } \\
\text { primaire }\end{array}$ & $\begin{array}{c}\text { De la lère } \\
\text { à la bè primaire }\end{array}$ & \\
\hline & \multirow[t]{2}{*}{ De 12 à 15 ans } & \multirow{2}{*}{$\begin{array}{c}\text { Enseignement } \\
\text { secondaire } \\
\text { de premier } \\
\text { degré }\end{array}$} & \multirow[t]{2}{*}{$\begin{array}{l}\text { lère et } 2 \grave{e} \\
\text { secondaire }\end{array}$} & $\begin{array}{l}\text { Section de transition : "Humanités générales } \\
\text { et technologiques" }\end{array}$ \\
\hline & & & & $\begin{array}{l}\text { Section de qualification: } \\
\text { «Humanités professionnelles et techniques " }\end{array}$ \\
\hline \multirow[t]{2}{*}{$\begin{array}{l}\text { Obligation } \\
\text { scolaire à } \\
\text { temps partiel }\end{array}$} & \multirow[t]{2}{*}{$\begin{array}{l}\text { De } 15 \text { à } \\
18 \text { ans }\end{array}$} & \multirow[t]{2}{*}{$\begin{array}{c}\text { Enseignement } \\
\text { secondaire } \\
\text { des second } \\
\text { et troisième } \\
\text { degrés }\end{array}$} & \multirow[t]{2}{*}{$\begin{array}{l}\text { De la 3è à } \\
\text { la bè } \\
\text { secondaire }\end{array}$} & $\begin{array}{l}\text { Enseignement à temps plein de transition } \\
\text { (général) ou de qualification (professionnel } \\
\text { ou technique) } \\
\text { Enseignement à temps partiel : " Enseignement } \\
\text { secondaire en alternance » dans un Centre } \\
\text { d'éducation et de formation en alternance } \\
\text { (CEFA) avec CISP ou CAI }\end{array}$ \\
\hline & & & & $\begin{array}{l}\text { Formation reconnue comme répondant aux } \\
\text { exigences de l'obligation scolaire } \\
\text { (ex. Apprentissage des Classes Moyennes) }\end{array}$ \\
\hline
\end{tabular}

Dans l'enseignement à temps partiel, des périodes de cours alternent avec une formation en entreprise. Les jeunes doivent y obtenir un contrat. II peut s'agir principalement d'une convention d'insertion socioprofessionnelle (CISP) ou d'un contrat d'apprentissage industriel (CAl) (cf. encadré 2).

La convention d'insertion socioprofessionnelle est conclue, pour 12 mois maximum, entre un Centre d'éducation et de formation en alternance (CEFA), une entreprise et un élève. Elle a pour objet d'organiser l'alternance entre une formation en entreprise et une formation dispensée par le CEFA. L'élève reçoit une indemnité mensuelle progressive à la charge de l'employeur qui représente entre $40 \%$ et $58 \%$ du Revenu minimum mensuel moyen garanti (1 1 17,88 € brut). L'apprentissage des Classes Moyennes constitue une voie parallèle à l'enseignement à horaire réduit en CEFA. Ce contrat a pour objet l'apprentissage d'une profession susceptible d'être exercée comme indépendant lartisanat, petite entreprise, etc.). L'apprenti doit suivre l'apprentissage pratique chez un indépendant et des cours de connaissances générales et professionnelles à I'Institut de formation permanente pour les PME. II perçoit une allocation mensuelle progressive variant de $200 €$ à $375 €$. Ces trois types de contrats visent ainsi le même public (jeunes de 15/16 à 18 ans) et consistent en une alternance entre une école et une entreprise.

À partir d'une approche sociologique de l'identité sociale, empruntée aux travaux de Dubar, nous tenterons de concevoir les politiques d'emploi destinées aux jeunes, à travers une perspective visant à appréhender la politique telle qu'elle se conçoit et telle qu'elle est pratiquée concrètement par les acteurs (Muller, 1998). Il s'agira ainsi d'illustrer comment les politiques publiques peuvent faire l'objet de réappropriation de la part des acteurs de terrain, comment elles se traduisent en termes de parcours d'emplois et de formes identitaires et, partant, quels enjeux elles laissent entrevoir ; enfin, quelles stratégies elles mobilisent en réinterpellant l'ensemble d'un dispositif public plus large de régulation de l'emploi.

\section{UNE PÉNÉTRATION DU CHAMP ÉDUCATIF}

Au début des années 1980 ( $c f$. encadré 4), l'enjeu de la formation professionnelle se déplace clairement 
dans le champ de l'éducation. Pour La Fédé, c'est ce dernier qu'il s'agit désormais de pénétrer, sans quoi la formation risquerait de s'éloigner davantage de l'activité professionnelle. Cette association patronale décide alors de prendre l'initiative de la production des qualifications qui lui sont nécessaires, en misant, notamment, sur la politique d'apprentissage industriel.

En 1985, alors que l'apprentissage industriel est réglementé depuis deux ans par la loi, le ministre de l'Emploi et du Travail s'inquiète de son manque d'application. La Flandre recourt davantage que la Wallonie à ce type de dispositif. Des divergences considérables entre le Nord et le Sud du pays dans le domaine de l'enseignement à horaire réduit se font jour (MET, 1999). La Fédé décide alors de prendre l'initiative en lançant les premières formules d'apprentissage industriel en Wallonie, en collaboration avec l'enseignement à temps plein. Ce dernier intervient alors. Il compte sur l'appui de La Fédé et des centres de formation pour trouver des entreprises accueillant des jeunes en stage de septième professionnelle. Les entreprises émettent cependant une condition importante : avoir un droit de regard sur le contenu même des formations. En effet, les formations proposées et organisées par les écoles ne répondent pas à leurs exigences (nouvelles technologies, commande numérique, etc.). La Fédé poursuit bien une stratégie de pénétration du champ éducatif, ressentie comme une véritable intrusion par le monde enseignant. Ainsi le cahier des charges de la formation est-il élaboré par l'entreprise qui oriente l'école dans le choix des formations à dispenser. Elle peut également choisir le rythme d'alternance lui convenant le mieux. La fédération met ainsi en avant l'avantage pour l'employeur de devenir «maîtres d'œuvre » de la formation dans le cadre du Contrat d'apprentissage industriel. L'école tente de s'adapter efficacement aux exigences de l'entreprise.

Ainsi, nous voyons combien l'apprentissage s'inscrit dans une politique à visée stratégique pour le monde patronal et syndical de cette branche professionnelle. Politique que l'on peut observer au début des années 1980, dans une conjoncture particulière de recommandations européennes, de problèmes d'emploi et de prolongation de la scolarité obligatoire. De ce face-à-face entre système productif et système éducatif, l'entreprise sort renforcée : au final, on

\section{Encadré 4}

\section{Contexte d'émergence du dispositif d'apprentissage industriel}

Le contrat d'apprentissage industriel est créé en juillet 1983, dans un contexte de chômage des jeunes notamment. II s'agit alors de lutter contre ce fléau. En Belgique, les systèmes de formation et d'insertion se voient désormais dénoncés en comparaison au "système dual » allemand. Celui-ci renvoie à l'existence et à l'articulation de deux lieux de formation : I'entreprise formatrice et l'école professionnelle complémentaire à temps partiel (Oerter, 1997). Mais sa transposition à la Belgique, où le " tout à l'école » semble prévaloir sur l'articulation école/entreprise, n'est guère aisée. Les débats fleurissent alors dans le pays sur ce qui est perçu comme un "hiatus » entre éducation et emploi. Le système éducatif se voit remis en cause, notamment au niveau de la formation technique et professionnelle. En effet, ainsi que le note Maroy (1997b), dans les années 1960 et 1970, l'ensemble de l'enseignement technique et professionnel a eu tendance à s'autonomiser du système productif : I'enseignement technique s'est de plus en plus intégré à l'enseignement général. C'est donc dans un climat de réflexion et de comparaison européenne que l'apprentissage industriel voit le jour en Belgique. La baisse du chômage des jeunes par l'insertion via la formation en alternance constitue l'hypothèse principale de l'élaboration de cette mesure. Pensé au sein du Conseil national du travail, I'apprentissage industriel relève du ministère de l'Emploi et du Travail et non de celui de l'Éducation. Mal perçu par les enseignants qui refusent de voir apparaître un nouveau partenaire dans le champ de l'éducation, il est considéré par ces derniers comme une arme à la disposition des employeurs pour mettre l'enseignement au service de leurs intérêts économiques. 
perçoit combien elle est devenue le véritable « maître d'œuvre » de la formation; de même, l'apprentissage industriel a été édicté dans le champ du travail et de l'emploi (Conseil national du Travail - loi du ministère de l'Emploi et du Travail) et non dans celui de l'Éducation nationale ${ }^{3}$; enfin, l'initiative et l'activation du projet ont reposé sur une alliance entre partenaires sociaux et ministère de l'Emploi et du Travail, dans le cadre d'une coalition gouvernementale de centre-droite.

\section{L'APPRENTISSAGE D'UN MÉTIER EN ENTREPRISE}

\section{Un travail identitaire}

Il s'agit donc bien de situer les trajectoires des jeunes en situation d'apprentissage industriel au cœur d'une tension entre deux logiques : une logique que l'on pourrait qualifier "d'éducative» et une logique « productive ». En principe, ces deux logiques pourraient faire référence à des systèmes et à des dispositifs différents, s'appuieraient sur des acteurs publics et professionnels distincts et se déploieraient dans des

3 À cette époque, l'enseignement relevait des compétences nationales puis, avec les réformes successives de l'État belge, a été transféré à la Communauté française de Belgique. espaces spécifiques ${ }^{4}$. Nous verrons plus loin qu'elles tendent à se confondre dans l'expérience concrète des acteurs. Si l'on essaye de comprendre les politiques, telles qu'elles se conçoivent et se pratiquent (Muller, 1998 ; Commaille et Jobert, 1998), il apparaît que le jeune apprenti se situe au centre d'un dispositif public qui devrait transparaître à travers l'expérience de l'ensemble du public-cible. S'intéresser uniquement à l'école ou à l'entreprise reviendrait à exclure $a$ priori une des deux logiques. Il nous est donc apparu plus enrichissant d'essayer de comprendre l'apprentissage industriel à partir des récits des jeunes qui ont à vivre et à articuler ces deux logiques. Ainsi formuleronsnous deux hypothèses. D'une part, les entretiens menés auprès des apprentis peuvent constituer un bon indicateur du fonctionnement global du système de l'apprentissage industriel au sein de La Fédé. D'autre part, si l'identité, telle que la conçoit $\operatorname{Dubar}^{5}$ (1992, 1996, 1998), consiste bien en un travail qu'accomplit l'acteur, ce travail identitaire prend toute son importance dans des situations où précisément des acteurs (les apprentis en l'occurrence) doivent articuler des logiques d'action diverses, voire contradictoires.

\footnotetext{
${ }^{4}$ Écoles et institutions de formation pour la première, entreprises et marché du travail pour la seconde.

${ }^{5}$ Identité duale composée de l'identité pour soi et de l'identité pour autrui. La socialisation rend possible la relation entre deux processus : biographique (Soi, sa trajectoire) et relationnel (Autrui, ses espaces de reconnaissance).
}

\section{Encadré 5}

\section{Une étude de cas}

Nous avons étudié des listes de formations en cours en septembre-octobre 1999, au sein d'une province de Belgique francophone caractérisée par un important héritage industriel. Nous avons noté le faible effectif de jeunes sous contrat d'apprentissage dans cette branche professionnelle, pourtant leader dans l'utilisation de ce dispositif. En effet, 29 personnes étaient concernées par ces mesures. Après avoir éliminé de la liste les apprentissages achevés (notre enquête portait avant tout sur des processus d'apprentissage en cours) ainsi que les abandons, nous avons finalement rencontré l'ensemble de la population recensée, à savoir 18 apprentis qui furent tous interviewés par entretiens semi-directifs. Afin d'élargir le cadre de l'analyse et d'apprécier la portée du phénomène étudié, l'enquête a également porté sur les formateurs, les responsables de formation en alternance (Institut de formation postscolaire de la branche professionnelle - IFPM - et Centre d'éducation et de formation en alternance) et les responsables de La Fédé; 28 personnes furent interrogées. Ces entretiens nous permettent de comprendre la politique d'apprentissage de cette branche professionnelle actuellement développée en Belgique francophone. 
Nous allons donc tenter de saisir ce travail d'articulation à l'aide des résultats d'une recherche (Levêque, 2000) menée auprès de jeunes en situation d'apprentissage industriel ( $c f$. encadré 5).

Lâge de cette population de jeunes apprentis apparait à tout le moins surprenant. Contrairement à la situation prescrite et attendue, tous les apprentis rencontrés sont âgés de 20 à 24 ans, alors que la loi cible des jeunes entre $15 / 16$ et 18 ans. Par ailleurs, quasiment tous ont terminé l'enseignement secondaire et ont donc également atteint l'âge de la majorité. Ils ne sont plus soumis à l'obligation scolaire, même à temps partiel. Ces résultats peuvent surprendre, dans la mesure où nous pensions rencontrer des jeunes correspondant au public des Centres d'éducation et de formation en alternance (CEFA), c'est-à-dire des jeunes en décrochage scolaire. Maroy (1997a, p. 12) confirme que la filière de l'enseignement à horaire réduit demeure réservée aux élèves en difficulté.

\section{La qualification plutôt que l'insertion}

Si l'on poursuit l'analyse, on remarque encore que la plupart des apprentis sont titulaires d'un diplôme de l'enseignement secondaire général. Les apprentis rencontrés bénéficient donc, avant la formation, d'un capital culturel, «concrétisé par les titres scolaires » (De Coster, 1996), plus élevé que le profil attendu ${ }^{6}$. Comment expliquer une telle situation?

La Fédé privilégie la qualification plutôt que l'insertion. La démarche consiste à partir de l'entreprise et de ses besoins et à organiser la formation selon ces derniers. La priorité n'est pas de trouver une entreprise d'accueil au jeune du $\mathrm{CEFA}^{7}$... En fait, la dynamique en cours semble bien se vouloir une réponse aux entreprises en manque de main-d'œuvre qualifiée. Favorisant avant tout une logique de production, elle ne souhaite pas entrer dans une mission de réin-

\footnotetext{
${ }^{6}$ Ces résultats entrent partiellement en contradiction avec ceux de Francq, Barré et Leloup (2000). Ces auteurs concluent qu'une minorité d'apprentis a suivi une scolarité dans le général (1,6\%). Ils seraient en revanche beaucoup plus nombreux à posséder un diplôme des filières techniques ou professionnelles.

${ }^{7}$ La mission principale des CEFA relève de l'insertion socioprofessionnelle des jeunes en difficulté (décret de l'exécutif de la Communauté française du 3 juillet 1991 organisant l'enseignement à horaire réduit, modifié par les décrets des 18 mars 1996, 24 juillet 1997, 17 juillet 1998 et 4 janvier 1999). Leur but est de placer le
}

sertion sociale de jeunes en décrochage scolaire, là n'est pas son objectif premier. Ainsi que l'avançait un responsable de La Fédé interviewé, « les 16 à 18 ans en difficulté, on en a eu dans quelques expériences et c'est extraordinairement difficile. Quand quelqu'un vient avec un bracelet à clous et une mèche orange, il n'entre pas dans l'usine. Ils n'ont même pas le minimum pour arriver à l'heure. L'entreprise n'est pas équipée pour ça. Et même quand ils ont quitté le système scolaire, s'ils sont disponibles pour un apprentissage, c'est que quelque chose ne marche pas ou n'a pas marché. C'est un public à risques, en dehors de nos moyens ». Selon Maroy (1997a, p. 13), les CEFA sont d'ailleurs confrontés à la difficulté de trouver des partenaires économiques disposés à supporter les coûts d'accueil et de formation des jeunes ${ }^{8}$. En précisant ses attentes auprès de jeunes diplômés de l'enseignement secondaire, La Fédé évite des problèmes comportementaux, de discipline, d'intégration difficile en usine. Elle opère une véritable sélection des publics afin d'obtenir la maind'œuvre qualifiée répondant à ses besoins. Cette sélection privilégie les jeunes de plus de 18 ans, possédant, à ses yeux, un certain bagage culturel, assez de maturité et la capacité d'apprendre. En effet, à l'école, ils ont appris à apprendre en suivant la filière générale. La Fédé va alors les former au métier. Concrètement, cette sélection s'opère par le ciblage du public. En effet, dans la majorité des cas, le Service public wallon de l'emploi et de la formation

plus de jeunes possible en entreprise : étape essentielle à leur insertion (Met, 1999, p. 92).

${ }^{8}$ Quelques entretiens menés auprès de conseillers en formation abondent dans ce sens : "Le public CEFA, il faut d'abord les éduquer. Et si on vise l'insertion, on leur fait faire des tâches subalternes, ils ne se qualifient pas et l'entreprise ne les garde pas à la fin de la formation. " (Conseiller à la formation en alternance de l'IFPM). "Ils se font renvoyer des stages parce qu'il y a des contraintes (se lever tôt, arriver à l'heure, respecter le matériel, les horaires...) ou alors ils s'y prennent mal avec les patrons » (Coordonnateur d'un CEFA). "Tandis que si on vise la qualification, tout le monde y gagne : le jeune est motivé et l'entreprise fait un investissement à long terme car elle pourra garder l'apprenti s'il a été bien formé " (Conseiller à la formation en alternance de l'IFPM). Le secteur a besoin de jeunes qualifiés mais l'école n'en forme pas suffisamment, c'est pourquoi « on se rabat sur les jeunes qui ont fait l'enseignement général. On ne trouve pas assez d'A2 ou de septième professionnelle » (Conseiller à la formation en alternance de l'IFPM). Cette réticence de la part des employeurs à engager des jeunes des CEFA explique sans doute le faible nombre de contrats d'apprentissage industriel en Belgique francophone ( $c f$. encadré 2). 
professionnelle (FOREM) ${ }^{9}$ envoie un courrier aux jeunes demandeurs d'emploi de la région répondant aux critères de La Fédé. Ceux-ci peuvent se limiter au fait de posséder un diplôme de l'enseignement général. "On est allé chercher des jeunes qui $n$ 'avaient pas d'orientation technique, qui avaient peut-être été dans le supérieur puis en échec... Mais avec leur bagage, ils ont une facilité d'assimiler les données plus abstraites et on a des résultats intéressants » (Responsable formation d'un syndicat de la branche). La Fédé considère que les élèves sortant d'une filière générale possèdent de meilleures bases théoriques facilitant leur apprentissage technique. Se voient ainsi de facto éliminés les jeunes sans diplôme de l'enseignement secondaire - voire même, dans beaucoup de cas, sans diplôme de l'enseignement

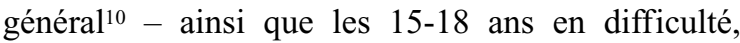
groupe-cible du dispositif. Les jeunes répondant positivement à ce courrier passent, par la suite, un test de connaissances dont la réussite conditionne l'entrée en contrat d'apprentissage.

Au niveau du diplôme obtenu, comme nous le savons, la plupart des apprentis ont suivi la filière générale dans le secondaire. Pour la majorité d'entre eux, le parcours scolaire s'est déroulé sans faille à ce niveau. La suite, en revanche, est marquée - à une exception près - par l'échec. L'enseignement supérieur, que la plupart d'entre eux ont tenté de poursuivre, s'est soldé par un abandon en cours d'études. Toutefois, le projet d'acquérir une formation demeure très présent. Ainsi, plusieurs apprentis poursuivent une autre formation en cours du soir.

\section{Une quête de reconnaissance sociale}

Une autre caractéristique de cette population est la forte présence de l'entourage familial, puisque plus de la moitié d'entre eux vivent toujours chez leurs parents. L'ensemble des entretiens menés témoignent de l'importance objective du milieu familial, présent pour les orienter et les soutenir. Ils ne se trouvent donc pas en situation objective d'exclusion ou de « galère »... Remarquons cependant que les jeunes

\footnotetext{
${ }^{9}$ Équivalent de l'Agence nationale pour l'emploi.

${ }^{10}$ Seuls 5 sur les 18 interviewés ont suivi un enseignement technique ou professionnel.
}

diplômés de l'enseignement général apparaissent aujourd'hui en rupture avec le projet familial initial et les desseins de leurs parents. Toutefois, l'expérience de l'apprentissage atteste de la reconstruction d'un nouveau projet. Et la famille finit par y adhérer. Ainsi, de nombreux parents ont manifesté un certain regret de voir leur enfant échouer dans l'enseignement supérieur, et plus encore prendre la décision de ne pas continuer dans la voie dans laquelle il s'était engagé. En ce qui concerne la situation d'apprentissage actuelle, ils font toutefois part de leur satisfaction, qui va de pair avec une reconnaissance de ce que leur enfant accomplit. Cette reconnaissance familiale est liée à la reconstruction d'un nouveau projet autour d'un travail gratifiant effectué lors de l'apprentissage.

Ce projet s'élabore autour du métier, reconnu et perçu comme lieu d'articulation associant formation et acquisition d'un travail valorisant. Au départ, les jeunes avaient une image négative de l'enseignement technique. Elle évolue vers une représentation plus positive et plus réaliste puisque l'apprentissage leur offre l'opportunité d'apprendre tout en découvrant un travail qu'ils valorisent fortement. "J'avais une mauvaise image du technique, comme tout le monde. En fait, on mélange technique et professionnel. On a l'impression que c'est la basse classe. En fait, c'est pas du tout ça. C'est une façon différente d'évoluer dans ses études; ça vaut pas moins que du général, c'est pas la même chose, c'est tout 》 (Bruno). «C'est quand on rate en général qu'on va en technique et puis c'est mal fréquenté, voilà ce que je me disais. Mais en fait, y a beaucoup de gens qui ont fait de belles carrières en ayant fait le technique au départ» (Raymond). "Ceux du technique et du professionnel, c'est les bêtes, ceux qui ont pas réussi en général. Mais mon image a changé. Ils savent faire quelque chose de leurs mains, c'est pas les bêtes finalement » (Xavier). "En technique, c'est des gens pas très recommandables. Quand on sort du primaire, on se dit que c'est pas bien» (Alexandre). "Je croyais que ceux qui travaillaient pas bien on les mettait là parce que c'est plus facile. Mais mon image a changé maintenant. On y apprend un métier » (Pascal). «J'croyais que c'était sale mais en fait, j'ai les mains sales deux fois par jour quand je vais chercher une pièce, sinon elles sont propres" (Adrien). "C'est vrai que c'est des gamins de merde qui sont là mais en fait, ouvrier, c'est pas péjoratif. Les gens s'en rendent compte quand je raconte ce que je fais » (Georges). 
Ainsi, lorsque, au cours des entretiens, les jeunes sont invités à parler de leur expérience de formation, celleci est, à chaque reprise, spontanément évoquée à partir de l'espace de l'entreprise. L'école n'est généralement abordée qu'après des relances de l'interviewer. Comment expliquer cette valorisation du lieu de travail par rapport à l'espace de formation ${ }^{11}$ ? Tout d'abord, ils considèrent l'entreprise comme le véritable lieu d'apprentissage d'un métier. Selon eux, le terrain fournit plus d'expériences concrètes, l'école étant perçue comme un lieu d'acquisition des connaissances de base. De plus, ils sont rémunérés pour les heures qu'ils effectuent en entreprise ${ }^{12}$. Et la dimension pécuniaire, fortement privilégiée par La Fédé13 constitue un élément clé de l'apprentissage industriel, pour attirer et motiver les jeunes. Il s'agit en fait d'une volonté du secteur de maintenir l'ancienne formule de rémunération plus avantageuse pour les jeunes et de leur octroyer une prime en fin d'année pour les motiver davantage. Les relations des jeunes avec les autres travailleurs sont généralement bonnes. Ils se sentent considérés comme de véritables collègues. Les autres ouvriers leur expliquent parfois comment accomplir telle ou telle tâche ou leur donnent des trucs de métier. Les contremaîtres ou chefs d'atelier les considèrent en général comme des ouvriers comme les autres. Ainsi, les entretiens avec les jeunes semblent refléter la mise en place d'un dispositif public d'apprentissage dans lequel l'entreprise apparaît comme

\footnotetext{
${ }^{11}$ Ils s'expriment en ces termes. Quand ils nous parlent de formation, il s'agit de la partie organisée à l'école tandis que, pour eux, l'entreprise est un espace de travail.

${ }^{12}$ Considéré comme un travailleur à temps partiel, l'apprenti reçoit une indemnité mensuelle d'apprentissage qui couvre les formations pratique et théorique $(536,50 €)$. Elle progresse avec l'avancement du stage et est calculée en fonction d'un pourcentage du salaire horaire minimum sectoriel sur la base de 37 heures par semaine $(7,25 €)$. Elle est limitée aux heures effectuées dans l'entreprise. Il faut cependant relever que l'apprenti reçoit une bourse d'un montant de $595 €$ en fin d'année s'il a suivi assidûment la totalité de la formation théorique et pratique.

${ }^{13}$ Initialement, la loi de 1983 prescrivait le paiement à l'apprenti d'une indemnité calculée selon un pourcentage du salaire minimum du travailleur dont on vise la qualification, conformément au barème salarial d'application dans la branche d'activité concernée. Toutefois, cet article s'est vu totalement modifié par la loi du 6 mai 1998 qui prescrit le calcul de l'indemnité sur la base d'un pourcentage du revenu mensuel moyen minimum garanti national, tel que fixé pour les travailleurs de 21 ans $(1186,31 €)$. La Fédé a décidé de maintenir le système initial durant le moratoire de deux ans, fixé par un arrêté du 19 août 1998, pour éviter de diminuer le montant de l'indemnité d'apprentissage.
}

principal espace de reconnaissance. Les savoirs professionnels caractéristiques de la socialisation professionnelle s'y transmettent principalement, l'école enseignant les savoirs de base. À cet égard, l'école n'apparaît nullement comme un espace socialement dévalorisé, avec toutefois certaines nuances en fonction de la formation suivie, de l'établissement ainsi que des secteurs. Au contraire, les apprentis y apprécient l'ambiance ; ils comparent la vie à l'école à celle qu'ils ont connue au moment où ils entreprenaient leurs humanités générales.

Toutefois, entre la fin de leurs études et l'entrée en apprentissage, tous ont vécu une première confrontation au marché du travail. Celle-ci, ainsi que le relève Dubar (1996, pp. 120-121), constitue un moment essentiel de la construction identitaire. Pour les apprentis, elle est aujourd'hui relatée en termes d'échecs ; cette expérience est confirmée plus globalement par Guillaume et Nandrin lorsqu'ils observent combien, en Belgique, «les diplômés du secondaire général connaissent grosso modo les délais d'insertion professionnelle les plus élevés». Toutefois, ils ajoutent "qu'au cours des années quatre-vingt, la détérioration des conditions d'insertion des jeunes diplômés est générale, quel que soit le niveau de leur formation. Seuls les diplômés de l'enseignement supérieur paraissent plus protégés » (Guillaume et Nandrin, 2000, p. 284). Sortis du secondaire ou d'études supérieures non achevées, sans expérience dans le monde du travail, ils n'ont pu s'y intégrer. En nous appuyant sur les travaux de Vernières (1993; 1997), nous pouvons formuler l'hypothèse que la formation scolaire doit généralement être assortie d'une expérience pratique et d'un minimum d'expérience au travail pour transformer en qualification ${ }^{14}$ les acquis issus de la formation. L'apprentissage industriel, dans le cas qui nous préoccupe, offrirait l'occasion d'une acquisition de cette expérience au travail. Les apprentis y intègreraient des savoirs techniques mais aussi organisationnels et relationnels.

\footnotetext{
${ }^{14}$ Sur les différentes significations du concept de qualification, on consultera notamment Dubar $(1987$; 1996). L'auteur relève trois sens : qualification individuelle, capacité à exercer un certain type de travail, acquise au terme d'un processus de formation et généralement sanctionnée par un diplôme ; qualification de l'emploi, liée au poste de travail tel qu'il est exercé dans l'entreprise ; classification salariale, position occupée dans une grille salariale, liée ou non à une convention collective (Dubar, 1987, pp. 139-140).
} 
Dès lors, les résultats peu favorables de leurs premières tentatives sur le marché du travail les ont conduits à accepter l'apprentissage industriel en vue d'acquérir une expérience qualifiante ainsi qu'un autre diplôme pour préparer leur deuxième face-àface avec le marché de l'emploi. Et ce, d'autant que cette seconde confrontation est envisagée sereinement (connaissance des débouchés, perspectives d'emplois, connaissance du fait objectif que $90 \%$ des apprentis sont engagés en fin de formation, etc.). Aux yeux des apprentis, l'entreprise apparaît comme un espace d'identification prioritaire, tant ils perçoivent qu'ils ne sont pas là pour réaliser les basses besognes mais bien pour transformer les acquis théoriques issus de l'école en compétences opératoires sur le terrain. Une fois de plus, le fait que la qualification soit au centre de cette expérience rejoint l'organisation de l'ensemble du dispositif mis en place par La Fédé.

\section{Une identité de métier}

Les jeunes apprentis accomplissent un véritable « travail identitaire » (Dubar, 1996) au cœur d'une tension entre deux logiques objectives (éducative et productive) ancrées sur le dispositif public d'apprentissage industriel. Ce travail identitaire ressort du discours des jeunes quand ils évoquent leur « apprentissage d'un métier en entreprise ». Il consiste en l'articulation de ces deux logiques, selon deux modalités biographiques (continuité ou rupture, selon la filière suivie dans l'enseignement secondaire), dans l'espace de reconnaissance qu'offre l'entreprise. Ces deux logiques potentiellement tensionnelles sont mises en perspective par le jeune grâce à la notion de métier, véritable pivot autour duquel il donne du sens au parcours d'emploi entrepris. À ses yeux, c'est autour de l' " apprentissage du métier » que finissent par se confondre logique éducative et logique productive.

À la lueur des travaux de Dubar (1992, 1996, 1998), ce travail identitaire peut se visualiser à l'aide de deux trajectoires - qui divergent, notamment, selon les filières d'enseignement poursuivies en secondaire accomplies par les jeunes depuis leur premier contact avec le marché du travail jusqu'à leur quête de reconnaissance au sein de l'entreprise par la recherche d'une identité de métier. Pour les jeunes qui se sont orientés vers l'enseignement général en humanités (environ trois quarts des jeunes), nous parlerons d'une identité de métier-découverte. En effet, ils ne se destinaient pas à un métier manuel au départ et ont découvert celui-ci pendant l'apprentissage industriel. En ce qui concerne les apprentis sortis de l'enseignement technique ou professionnel, nous parlerons d'une identité de métier-filière, puisque la filière technique ou professionnelle les a conduits vers l'apprentissage.

Non reconnus sur le marché du travail, les jeunes issus de la filière générale étaient en rupture tant par rapport à leur parcours scolaire que par rapport aux projets familiaux. Ils ont décidé d'interrompre leurs études supérieures pour se confronter au marché de l'emploi. Les apprentis issus de la filière technique ou professionnelle évoluaient, en revanche, en continuité avec leur filière d'enseignement et cherchaient, vainement, un emploi dans le domaine étudié.

Ces trajectoires attestent d'un « déplacement » identitaire effectué par les acteurs à travers une quête de reconnaissance au sein de l'entreprise. Elles témoignent également d'une volonté d'intégration de ces jeunes apprentis dans l'entreprise. À la suite de Joseph et Lochet (1999, pp. 8-9), nous pouvons conclure à une intégration par la formation en alternance, accompagnée d'une expérience professionnelle qualifiante. En effet, pour ces auteurs, l'intégration ${ }^{15}$ en entreprise nécessite une certaine pérennité de relation d'emploi ou une action conséquente de formation, conditions réunies au niveau de l'apprentissage industriel mis en place dans le cadre de La Fédé.

\section{UNE GESTION PAR LA DÉROGATION}

La perspective identitaire nous invite à réinterpeller le dispositif public de formation à la lueur de ce travail de déplacement identitaire effectué par les apprentis.

Les jeunes rencontrés possèdent tous, à une exception près, un diplôme de l'enseignement secondaire supé-

\footnotetext{
${ }^{15}$ Joseph et Lochet (1999, p. 6) définissent l'intégration en entreprise comme un "processus de construction d'une expérience professionnelle qualifiante et de reconnaissance de cette qualification à travers un certain type de relation d'emploi qui s'inscrit potentiellement dans le moyen-long terme $»$.
} 
rieur. Ils sont donc âgés de plus de 18 ans (20 à 24 ans). Rappelons que la loi prescrit un âge maximum de 18 ans en début d'apprentissage, sauf dérogation. Et c'est ici que ce terme prend toute son ampleur, dans la mesure où le secteur ne fonctionne quasiment que par dérogation admise par son Comité paritaire d'apprentissage. Idée que rejoint Mariën $(1997$, p. 7) lorsqu'il déclare combien chaque branche professionnelle applique une version « adaptée » de la réglementation de l'apprentissage industriel, indépendamment de la législation.

Cette pratique de la dérogation à l'âge permet une sélection des jeunes au service d'une logique de qualification privilégiée par les employeurs et les partenaires sociaux de la branche. Le principal enjeu pour La Fédé et les employeurs consiste à trouver des jeunes dotés de compétences qui leur permettront d'arriver en fin de formation d'une part et répondant aux exigences du métier et de l'entreprise d'autre part. La dérogation permet la sélection conduisant, elle, au taux d'engagement de $90 \%$ des apprentis. Les apprentis ainsi choisis bénéficient d'une véritable reconnaissance sur le lieu de travail. Ils constituent un réel investissement pour l'employeur.

Cette reconnaissance objective des jeunes par l'entreprise atteste d'un réel besoin de cette dernière en matière de qualification plutôt que d'une mission de réinsertion de jeunes en difficultés. Les entreprises de La Fédé recherchent bel et bien une main-d'œuvre qualifiée qu'elles ne trouvent pas aisément à la sortie de l'école ; elles adoptent donc une réelle démarche de sélection du public à former. Tout en privilégiant les étudiants diplômés du secondaire, elles dérogent systématiquement à la loi en faisant appel à des apprentis âgés de plus de 18 ans. Le Comité paritaire accepte en fait qu'ils aient atteint 25 ans en fin d'apprentissage. Les CEFA n'acceptant pas de jeunes de plus de 21 ans, on comprend encore, par le recours systématique à la dérogation, combien ces derniers ne sont plus les partenaires pédagogiques prioritaires et sont littéralement contournés. En effet, la plupart des jeunes recrutés ne sont plus soumis à l'obligation scolaire ; il ne s'agit donc pas d'un public en décrochage dans l'enseignement à horaire réduit (CEFA) mais bien de jeunes demandeurs d'emploi diplômés depuis peu du système scolaire.

Ainsi, dans tous les cas, l'application de cette dérogation à l'âge entraîne de facto l'exclusion du groupecible du système d'apprentissage tel qu'il est présenté au niveau institutionnel. Ce dispositif s'organise à distance de l'enseignement à horaire réduit et des jeunes, entre 15/16 et 18 ans, fréquentant l'école à temps partiel. De simple possibilité offerte aux metteurs en œuvre de cette politique, la dérogation se voit systématiquement appliquée. L'exception devient ainsi la règle.

Si l'on tente de synthétiser le système d'action qui se dégage progressivement autour de La Fédé, nous pouvons retenir que pour répondre au défi majeur que représente la qualification de sa main-d'œuvre, La Fédé opte pour une série de stratégies visant à attirer et à sélectionner les jeunes dans son giron : pratiques de sélection, d'attraction par la rémunération, motivation par l'emploi et les perspectives d'avenir, valorisation et reconnaissance de l'apprenti, acquisition d'un métier, formation, séduction par des informations publicitaires. Ce faisant, tout en acceptant d'aider les établissements d'enseignement à trouver des stages d'accueil pour leurs élèves, elle développe une stratégie de pénétration du champ éducatif afin de déterminer les programmes d'apprentissage ; ce qui constitue en soi une remise en question du système d'enseignement tel qu'il est organisé aujourd'hui par la Communauté française de Belgique. Pour mener à bien ces stratégies autour de la qualification, La Fédé développe des alliances avec ses partenaires au sein des différentes instances auxquelles elle participe avec ses représentants patronaux (FOREM ${ }^{16}$, IFPM $^{17}$, Comité Paritaire). Un véritable système d'action se dégage ainsi pour produire le dispositif public d'apprentissage orienté vers les futurs jeunes apprentis ; dispositif dont les effets se lisent à travers le travail identitaire et de construction de sens qu'ils entreprennent. Pour réguler cet ensemble et répondre à l'impératif entrepreneurial de sélection de la maind'œuvre, La Fédé recourt à la dérogation, véritable outil de régulation publique.

À travers l'exposé de ce cas, on observe que l'acteur entrepreneurial ne vise pas prioritairement - comme beaucoup le pensent en Belgique - une dérégulation des politiques publiques mais participe davantage à un exercice d'activation discrétionnaire des poli-

\footnotetext{
${ }^{16}$ Service public wallon de l'emploi et de la formation professionnelle (équivalent de l'Agence nationale pour l'emploi).

${ }^{17}$ Institut de formation postscolaire de la branche professionnelle
} étudiée. 
tiques de l'emploi. La régulation par la dérogation exprime avant tout une mobilisation de moyens en fonction des intérêts sectoriels et conjoncturels des entreprises de La Fédé : celles-ci, en quête de maind'œuvre qualifiée, apprennent à solliciter les jeunes en les soumettant à une épreuve de sélection. Par ailleurs, «l'expression identitaire » de cette politique menée auprès de jeunes fortement motivés par le dispositif, nous permet également de comprendre le taux d'engagement de près de $90 \%$ des apprentis ayant achevé leur apprentissage ! La reconnaissance de ces jeunes autour d'identités de métier ne prend ici pleinement sens qu'à la lueur du dispositif public régulé par le recours systématique de La Fédé à la dérogation.

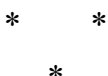

La politique sectorielle de formation de La Fédé est sous-tendue par un besoin en main-d'œuvre qualifiée des entreprises. Il constitue le point de départ de l'apprentissage. Se trouvant dans l'impossibilité de sélectionner des individus déjà socialisés par le système scolaire, La Fédé choisit d'organiser ellemême cette socialisation en entreprise en recourant à des stratégies de sélection (jeunes de plus de 18 ans, issus du secondaire général et ayant réussi les tests) sous-tendues par un recours systématique à la dérogation à l'âge à l'entrée en apprentissage. Le jeune est alors professionnalisé dans l'esprit et les normes propres à l'entreprise ; selon Maurice, Sellier et Silvestre (1982, p. 284) cela contribue à le rendre plus dépendant de l'entreprise et tend à adapter la professionnalité du travailleur aux exigences de son propre système de production. À la lueur des travaux de Joseph et Lochet (1999), l'apprentissage constitue un mode de régulation, interne à l'entreprise, de l'accès à ses postes de travail et à ses emplois.

Nous avons vu que les employeurs recouraient au dispositif en pénétrant de manière précoce le champ éducatif. En effet, ces entreprises en demande de main-d'œuvre qualifiée se voient contraintes de devenir des agents de socialisation parce que l'enseignement technique et professionnel fournit aujourd'hui trop peu de jeunes répondant aux exigences des métiers de la branche. Elle prendraient ainsi très nettement leurs marques à l'égard du système scolaire institutionnel (Tanguy, 1998), tel qu'il est organisé aujourd'hui par la Communauté française de Belgique. Dans le contexte actuel de restructuration des relations système productif/système éducatif, liée à l'exigence patronale d'adéquation immédiate des compétences du jeune au sortir de l'école (Maroy, 1997b), l'apprentissage industriel devient un moyen au service d'une gestion des ressources humaines de plus en plus flexible et individualisante.

Cette gestion par la dérogation apparaît comme une expression plus générale d'une véritable pratique de sélection de la main-d'œuvre afin d'en mieux discerner les compétences (Paugam, 2000). Le succès de l'apprentissage industriel (eu égard au pourcentage de jeunes engagés en fin de formation) peut se comprendre par le tri opéré parmi les jeunes. Tri qui reflète fondamentalement combien cette gestion s'opère par l'exclusion des jeunes ne rentrant pas dans les critères fixés par La Fédé... En effet, qui serait alors potentiellement exclu de ce dispositif public ? Le public-cible lui-même, pour lequel l'apprentissage industriel a été instauré en 1983, à savoir les jeunes entre 15/16 et 18 ans qui suivent un enseignement à horaire réduit dans un CEFA.

Cette mise à l'épreuve des jeunes apprentis illustre une tendance actuelle des entreprises à recourir à l'intérim ou aux contrats à durée déterminée (ministère de l'Emploi et du Travail, 2000) afin d'opérer une sélection au sein de la main-d'œuvre. À ce jeu, il apparaît que la probabilité d'accéder à un emploi stable augmente avec le niveau de qualification ; et ce, d'autant que notre approche identitaire révèle combien les jeunes apprentis semblent se mobiliser à travers l'ensemble du dispositif. Interrogés, ils n'ignorent pas cette situation duale du marché du travail, situation qu'ils ont, pour la plupart, personnellement vécue ou qu'ils connaissent à travers les récits de nombre de leurs copains en difficulté d'insertion. S'ils ne s'estiment plus directement concernés par cette situation de précarité sociale, ils continuent à en livrer les traces à travers leurs trajectoires identitaires et leurs quêtes de reconnaissance.

L'intérêt des acteurs entrepreneuriaux de La Fédé ne peut toutefois, à lui seul, être invoqué comme facteur explicatif de cette précarité entretenue à travers, notamment, la régulation par la dérogation. Notre cas d'analyse illustre combien l'entreprise nécessite souvent une main-d'œuvre qualifiée et rémunérée en 
conséquence. Par ailleurs, partie prenante du compromis social constitutif de l'État-providence, le patronat n'a guère intérêt à miser sur une précarisation généralisée de la main-d'œuvre ou sur une diminution des cotisations sociales. Au contraire, notre analyse suggère plutôt que nous n'assistons pas à une dérégulation des politiques publiques d'emploi mais plutôt à une activation discrétionnaire de celles-ci.
Ainsi, ce constat d'une gestion par la dérogation laisse apparaître un consensus tacite entre acteurs privés et puissance publique en vue de diminuer, ne fut-ce que faiblement, le chômage des jeunes, au prix d'une précarisation des acteurs les plus fragiles. La dualisation apparaissant en quelque sorte comme le prix à payer d'une activation des politiques publiques dont la gestion par la dérogation ne serait qu'une illustration.

\section{Bibliographie}

Brion R., Moreau J.-L. (1996), La Fédé, 50 ans au cour de l'industrie, Éditions Racine, Bruxelles.

Castel R. (1995), Les métamorphoses de la question sociale, Fayard, Paris.

Commaille J., Jobert C. (1998), Les métamorphoses de la régulation politique, LGDJ, coll. « Droit et société », Paris.

De Coster, M. (1996), Introduction à la sociologie, De Boeck Université, coll. « Ouvertures sociologiques », Bruxelles.

Dubar, C. (1992), « Formes identitaires et socialisation professionnelle », Revue Française de Sociologie XXXIII, pp. 505-529.

Dubar C. (1996) (1991), La socialisation. Construction des identités sociales et professionnelles, Colin, coll. «U $»$, Paris.

Dubar C. (1998) (1994), « Identités collectives et identités individuelles dans le champ professionnel », in De Coster M., Pichault F. (Eds), Traité de sociologie du travail. Préface d'Alain Touraine, De Boeck Université, coll. «Ouvertures sociologiques », Bruxelles, pp. 385-400.

Dubet F. (1994), Sociologie de l'expérience, Le Seuil, Paris.

Fitoussi J.-P., Rosanvallon P. (1996), Le nouvel âge des inégalités, Le Seuil, Paris.

Francq B., Leloup X., Barré P. (1998), « Profils de participation des entreprises et formation en alter- nance », Critique Régionale 26/27, pp. 121-130.

Francq B., Barré P., Leloup X. (2000), « La participation des entreprises à l'apprentissage industriel : production de qualifications techniques ou sociales ? », in Bajoit, G. et al. (Eds), Jeunesse et société, De Boeck Université, coll. " Ouvertures sociologiques », Bruxelles, pp. 245-258.

Giovannini N. (2000), « La politique sociale sous le sceau de l'État-providence actif : esquisse d'évaluation critique », Pyramides 1, pp. 157-169.

Guillaume J.-F., Nandrin H. (2000), « Les trajectoires d'insertion professionnelle des jeunes belges francophones ", in Bajoit, G. et al. (Eds). Jeunesse et société, De Boeck Université, coll. " Ouvertures sociologiques », Bruxelles, pp. 277-288.

Institut de Formation Post scolaire de La Fédé (1999), Document de présentation de l'apprentissage industriel à La Fédé, IFPM.

Joseph O., Lochet J.-F. (1999), « L'insertion dans l'entreprise des jeunes sortant de l'enseignement secondaire », Formation Emploi n ${ }^{\circ}$ 68, pp. 5-19.

Levêque A. (2000), L'identité : reflet d'un système. L'apprentissage industriel à la Fédé, Mémoire présenté en vue de l'obtention du grade de licenciée en sociologie de l'université de Liège.

Mariën L. (1997) (1993), «L'apprentissage de professions exercées par des travailleurs salariés », Revue du travail $\mathrm{n}^{\circ} 11$. 
Maroy C. (1997), « Vertus des formations en alternance et contexte sociétal », Reflets et perspectives, XXXVI, pp. 5-17.

Maroy C. (1997b), « Entre autonomie et assujettissement : genèse et enjeux de la recomposition des relations école/entreprise en Belgique francophone », $L a$ Revue Nouvelle 3, pp. 86-99.

Maruani M., Reynaud E. (1993), Sociologie de l'emploi, La Découverte, Paris.

Maurice M., Sellier F., Silvestre J.-J. (1982), Politique d'éducation et organisation industrielle en France et en Allemagne, PUF, coll. « Sociologies », Paris.

Ministère fédéral de l'Emploi et du Travail, (1999), La politique fédérale de l'emploi, Rapport d'évaluation 1999, Bruxelles, nov.

Ministère fédéral de l'Emploi et du Travail (2000), $L a$ politique fédérale de l'emploi, Rapport d'évaluation 2000, Bruxelles, nov.

Muller P. (1998), Les politiques publiques, PUF, coll. «Que sais-je?», Paris.

Oerter H. (1997), « Le système dual en Allemagne : une formation professionnelle par apprentissage », Reflets et perspectives XXXVI, pp. 35-53.
Paradeise C. (1988), "Les professions comme marché du travail fermé », Sociologie et sociétés XX (2), pp. 9-21.

Paugam S. (1991), La disqualification sociale. Essai sur la nouvelle pauvreté, PUF, Paris.

Paugam S. (2000), Le salarié de la précarité. Les nouvelles formes de l'intégration professionnelle, PUF, Paris.

Rosanvallon P. (1981), La crise de l'État-providence, Paris, Seuil.

Rosanvallon P. (1995), La nouvelle question sociale. Repenser l'État-providence, Le Seuil, Paris.

Tanguy L. (1998), « La formation, une activité sociale en voie de définition ? » in De Coster M., Pichault F. (Eds.), Traité de sociologie du travail. Préface d'Alain Touraine, De Boeck Université, coll. « Ouvertures sociologiques », Bruxelles, pp. 185212.

Vernières M. (1993), Formation-Emploi. Enjeu économique et social, Cujas, Paris.

Vernières M. (Ed.) (1997), L'insertion professionnelle. Analyses et débats, Économica, Paris.

\title{
Résumé
}

\section{La gestion par la dérogation : une politique d'apprentissage industriel en Belgique francophone}

\author{
par Audrey Levêque et Didier Vrancken
}

À travers l'étude d'un dispositif belge de formation en alternance, l'apprentissage industriel, et à partir d'une perspective sociologique de l'identité (Dubar), cet article tente d'appréhender la politique de formation-emploi mise en oeuvre par une des branches professionnelles industrielles les plus importantes en Belgique francophone. Nous illustrerons comment une politique publique peut faire l'objet de réappropriation de la part des acteurs de terrain, mettant ici en place une véritable gestion par la dérogation. Ainsi, en dérogeant systématiquement à la limite d'âge à l'entrée en formation, les metteurs en œuvre sélectionnent un public plus âgé correspondant davantage à leurs attentes que le groupe-cible du dispositif : les jeunes de 15 à 18 ans ayant quitté l'enseignement à temps plein. 